\title{
A NEW MIXED PRECONDITIONING METHOD BASED ON THE CLUSTERED ELEMENT-BY-ELEMENT PRECONDITIONERS
}

\author{
T.E. Tezduyar, M. Behr, S.K. Aliabadi, S. Mittal and S.E. Ray
}

\begin{abstract}
We describe a new mixed preconditioning method for finite element computations. In the clustered element-by-element (CEBE) preconditioning, the elements are merged into clusters, and the preconditioners are defined as series products of cluster level matrices. The (cluster companion) $\mathrm{CC}$ preconditioners are based on companion meshes associated with different levels of clustering. For each level of clustering, we construct a CEBE preconditioner and an associated CC preconditioner. Because these two preconditioners complement each other, when they are mixed, they give better performance. Our numerical tests, for two- and three-dimensional problems governed by the Poisson equation, show that the mixed CEBE/CC preconditioning results in convergence rates which are significantly better than those obtained with the best of the CEBE and CC methods.
\end{abstract}

\section{Introduction}

The element-by-element (EBE) preconditioners, which are constructed as series products of element level matrices, have been successfully applied to several classes of problems [1-4]. They can be used effectively with the conjugate-gradient and GMRES [5] methods, and are highly vectorizable and parallelizable (see $[3,6,7])$. They can also be used together with the implicit-explicit and adaptive implicit-explicit timeintegration schemes [4,7-9].

In the CEBE preconditioning $[10,11]$, the elements are merged into clusters, and the preconditioners are constructed as series products of cluster level matrices. In [10], the CEBE preconditioning was used for solving problems governed by the Poisson equation. In [11], the CEBE preconditioning was employed to solve compressible and incompressible flow problems. Applications to the space-time finite element formulations were included in [11]. To facilitate vectorization and parallel processing,

1991 Mathematics Subject Classification. Primary 65N30, 65M55; Secondary 65F35.

This research was sponsored by NASA-JSC under grant NAG 9-449, by NSF under grant CTS-8796352, and by ALCOA Foundation. Partial support for this work has also come from the Army Research Office contract number DAAL03-89-0038 with the Army High Performance Computing Research Center at the University of Minnesota.

Detailed version of this paper will appear elsewhere. 
as it is done in the grouped element-by-element (GEBE) method [6], the clusters can be grouped in such a way that no two clusters in any group are connected. Furthermore, depending on the number of elements in the cluster, within each cluster, elements can again be grouped in the same way. The number of elements in each cluster can be viewed as an optimization parameter that can be varied to minimize the computational cost. In fact, in [12], the unsteady incompressible flow computations were performed by using a space-time finite element formulation with a nearly optimal cluster size which was determined by numerical experimentation.

In the construction process of the $\mathrm{CC}$ preconditioners, we first start with a "primary" mesh with different levels of clustering. For each level of clustering in this primary mesh, we define a "companion" mesh, such that each cluster of the primary mesh forms an element of the companion mesh. We then define a CC preconditioner based on each companion mesh, such that there is a CC preconditioner associated with each CEBE preconditioner based on a certain level of clustering. This way, for each level of clustering, we obtain a CC preconditioner which we expect to have more intercluster coupling information then the associated CEBE preconditioner has. Conversely, the CEBE preconditioner can be expected to have more intra-cluster coupling information than the associated $\mathrm{CC}$ preconditioner has.

The mixed CEBE/CC preconditioning, first introduced by Tezduyar et al. [13], is based on the assumption that the CEBE and $\mathrm{CC}$ preconditioners complement each other, and therefore when they are mixed together they will result in better convergence rates. The mixed preconditioning is implemented by using these two preconditioners alternately at each inner GMRES iteration.

\section{CEBE (Clustered Element-by-Element) Preconditioning}

In the CEBE method the elements are partitioned into clusters. For example, Figure 1 shows four different levels of clustering for a uniform $16 \times 16$ mesh. The cluster boundaries are marked with thick lines. In the first frame each cluster consists of one element, and therefore this would lead to an EBE method. In the last frame the cluster size is $8 \times 8$; the next level of clustering after that (i.e., level 5) would lead to a direct solution method. The CEBE preconditioning is based on approximation of the global matrix by a sequential product of cluster level matrices. We can give two examples (see [10,11]): 2-Pass CEBE preconditioner and Crout CEBE preconditioner.

\section{CC (Cluster Companion) Preconditioning}

Consider a mesh with different levels of clustering. For each level of clustering in this "primary" mesh, we define a "companion" mesh, such that each cluster of the primary mesh forms an element of the companion mesh. For example, Figure 1 can now also be seen as showing the companion meshes associated with four different levels of clustering. In each frame of Figure 1, the thick lines not only mark the cluster boundaries for a certain level of clustering, but also depict the companion mesh associated with that level of clustering. In the first frame the companion mesh is the same as the primary mesh. In the last frame the companion mesh is a $2 \times 2$ mesh. The $\mathrm{CC}$ preconditioner associated with a certain CEBE preconditioner is constructed based on the companion mesh associated with the clustering the CEBE preconditioner is based on. The details can be found in [13]. 


\section{Mixed CEBE/CC Preconditioning}

The CEBE preconditioner has more intra-cluster coupling information than the CC preconditioner has. The $\mathrm{CC}$ preconditioner, on the other hand, has more inter-cluster coupling information. Therefore, it is reasonable to expect that when these two preconditioners are mixed together they lead to better convergence rates. We mix these two preconditioners by using them alternately at each inner GMRES iteration. Recently Saad [14] has formulated a new version of the GMRES algorithm which allows changing the preconditioner at every inner iteration. A GMRES subroutine, based on this new formulation and made available to us by Saad, is what we use to implement our mixed preconditioning.

In our notation, CEBE-L will represent the CEBE preconditioning based on clustering level $\mathrm{L}, \mathrm{CC}-\mathrm{L}$ will represent the $\mathrm{CC}$ preconditioning based on companion mesh $L$ associated with clustering level $L$, and CEBE/CC $-L$ will represent the mixing of the two. It is important to realize that, once it is decided what the level $L$ of our preconditioning is, there is no need for any intermediate level meshes. In fact, in the case of unstructured meshes, there is no intermediate meshes between the primary and coarse meshes. Figure 2 shows two hypothetical meshes superimposed on each other; a fine and a coarse one, both totally unstructured and arbitrary. In this case the clustering is achieved by distributing the elements of the fine mesh among the elements of the coarse mesh. Figure 2 also shows how a cluster of elements (marked with shaded areas) forms, in an approximate sense, an element of the coarse mesh.

\section{Numerical Tests}

We tested the preconditioners defined in Sections 2, 3 and 4 on several test problems governed by the Poisson equation; here we report those with zero forcing functions (for those with nonzero forcing functions, see [13]). For all the results reported here a Krylov space of dimension 20 was used and the initial guess for the solution vector was set to zero. We monitor the scaled residual during the inner iteration loop (number of inner iterations is the same as the size of Krylov space) for one outer iteration. Details of the test problems can be found in [13].

In the tests with two-dimensional non-uniform meshes, a cylinder of unit radius is located at the center of a $32 \times 32$ square, and the boundary conditions are non-symmetric. Figure 3 shows, for a $128 \times 32$ primary mesh, the convergence histories for $L=3$ and 4. The CEBE/CC preconditioners outperform the others. Results for a $256 \times 64$ primary mesh for $L=3$ and 4 are shown in Figure 4.

In the tests with three-dimensional uniform meshes, the domain is a rectangular parallelepiped, discretized by trilinear brick elements. Homogeneous Dirichlet type boundary conditions are imposed on five faces of the domain: On the sixth face, a boundary condition of bi-quadratic form, with a maximum value of 1 at the center and 0 at the edges is imposed. In Figure 5, we show, for a $128 \times 128 \times 64$ primary mesh, the convergence of the diagonal, CEBE-4, CC-4 and CEBE/CC-4. The CEBE/CC preconditioner achieves much higher rate of convergence than either of its two components. 


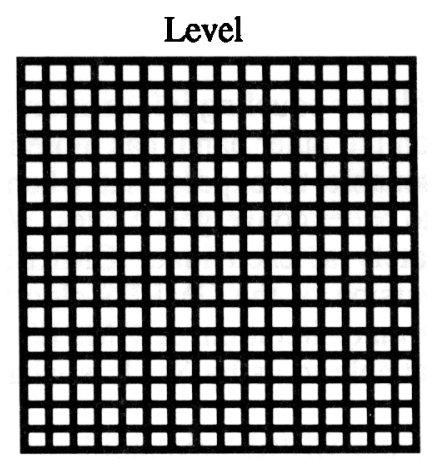

Level 3

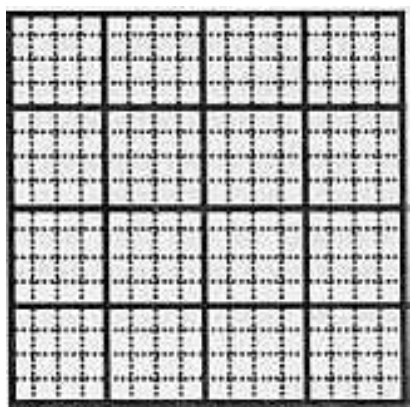

Level 2

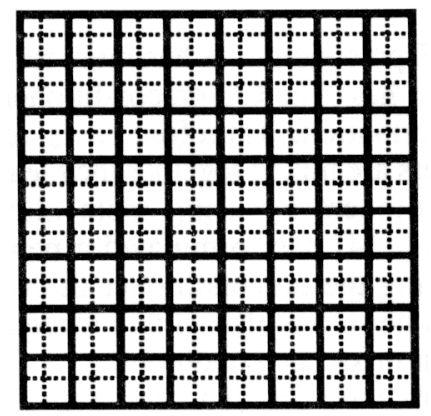

Level 4

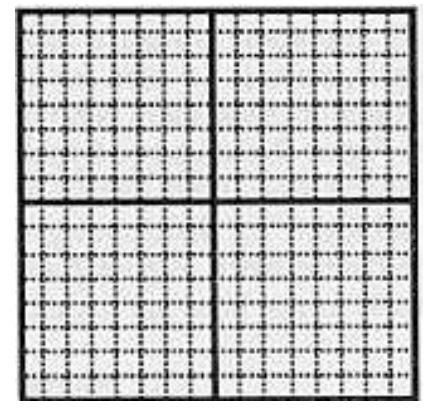

Figure Four different levels of clustering for a uniform $16 \times 16$ mesh; in each frame the thick lines depict the cluster boundaries and the associated companion mesh.

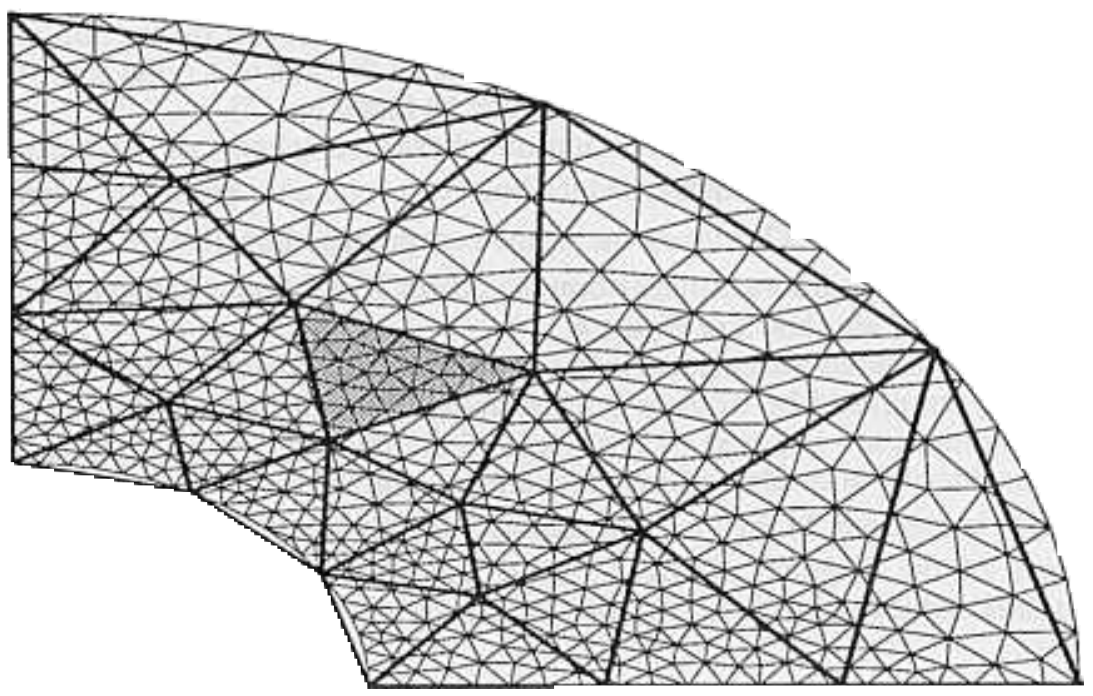

Figure 2. The CEBE/CC strategy for totally unstructured meshes: the fine (primary) and coarse (companion) meshes and the cluster of elements associated to a typical element of the coarse mesh. 
Level 3
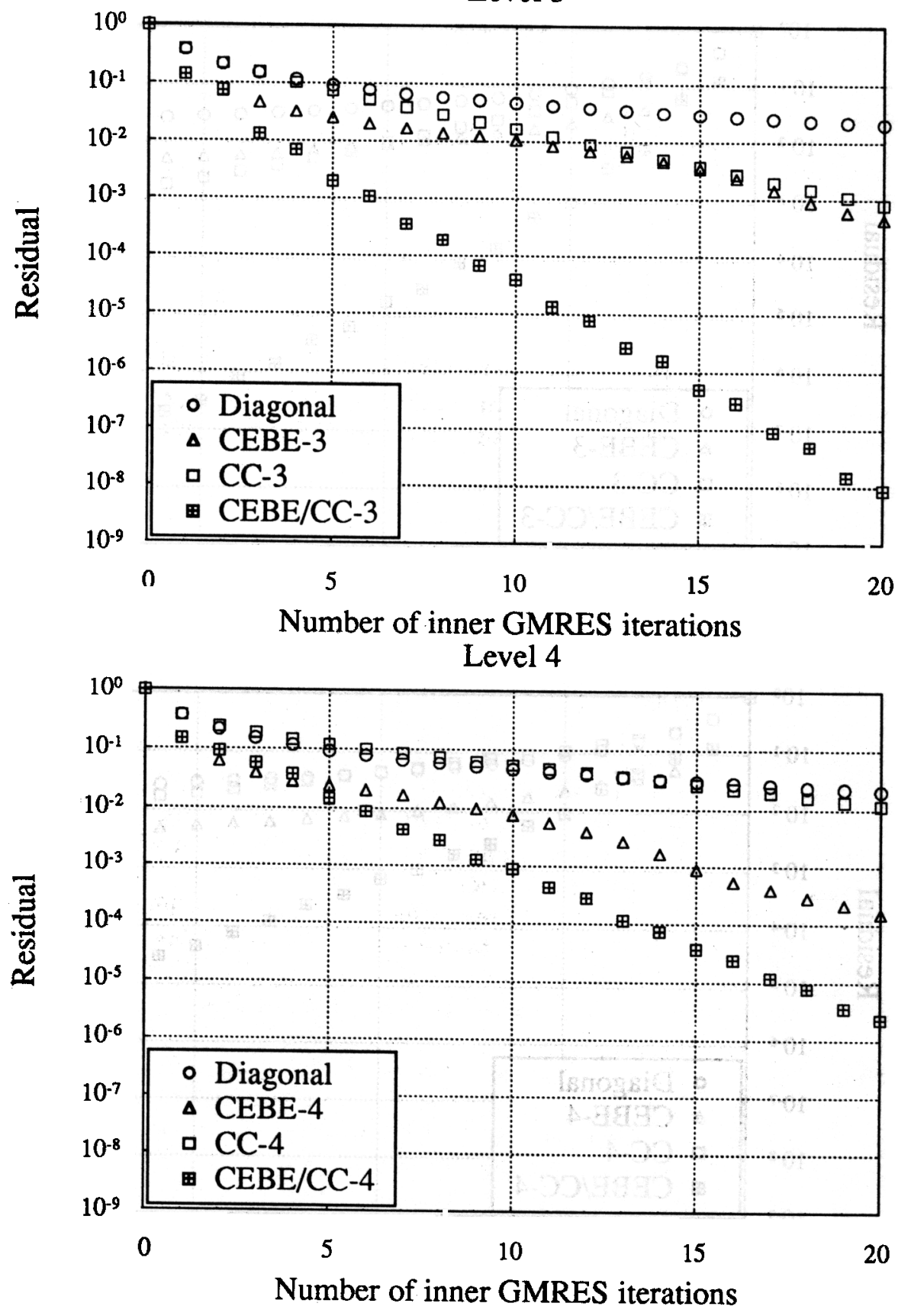

Figure 3. Convergence histories for a two-dimensional mesh of $128 \times 32$ elements. 
Level 3
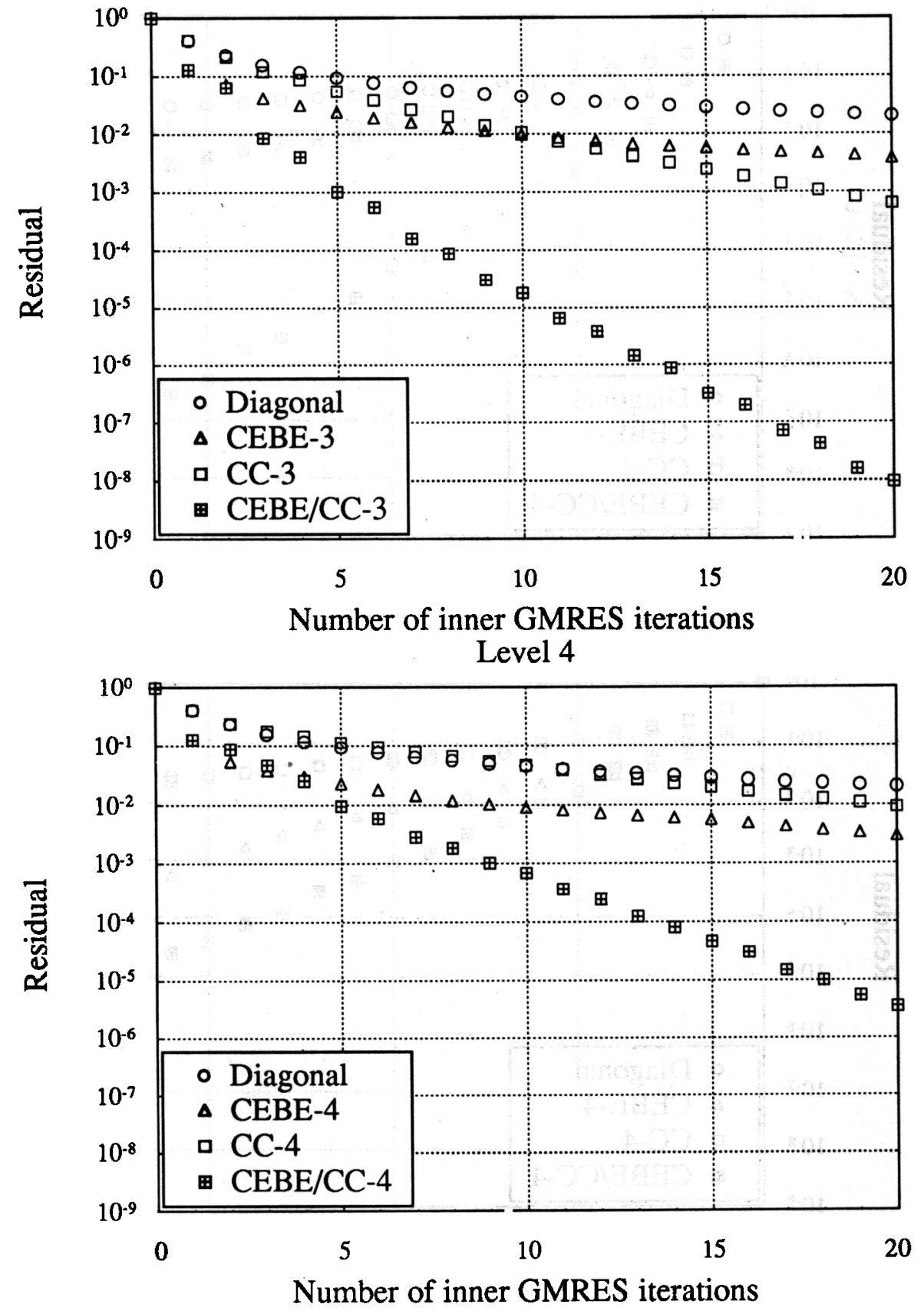

Figure 4. Convergence histories for a two-dimensional mesh of $256 \times 64$ elements. 
Level 4

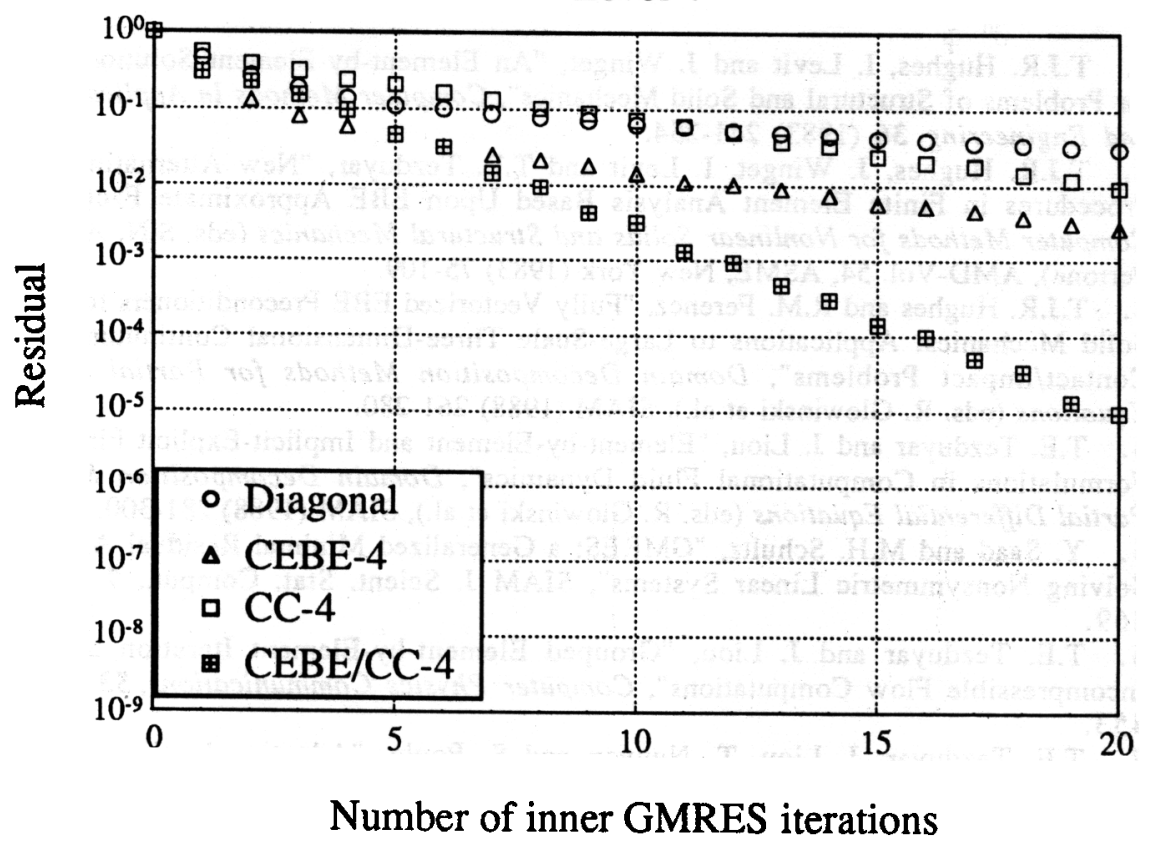

Figure 5. Convergence histories for a three-dimensional mesh of $128 \times 128 \times 64$ elements. 


\section{REFERENCES}

1. T.J.R. Hughes, I. Levit and J. Winget, "An Element-by-Element Solution Algorithm for Problems of Structural and Solid Mechanics", Computer Methods in Applied Mechanics and Engineering, 36 (1983) 241-254.

2. T.J.R. Hughes, J. Winget, I. Levit and T.E. Tezduyar, "New Alternating Direction Procedures in Finite Element Analysis Based Upon EBE Approximate Factorizations", Computer Methods for Nonlinear Solids and Structural Mechanics (eds. S.N. Atluri and N. Perrone), AMD-Vol. 54, ASME, New York (1983) 75-109.

3. T.J.R. Hughes and R.M. Ferencz, "Fully Vectorized EBE Preconditioners for Nonlinear Solid Mechanics: Applications to Large-Scale Three-Dimensional Continuum, Shell and Contact/Impact Problems", Domain Decomposition Methods for Partial Differential Equations (eds. R. Glowinski et al.), SIAM (1988) 261-280.

4. T.E. Tezduyar and J. Liou, "Element-by-Element and Implicit-Explicit Finite Element Formulations in Computational Fluid Dynamics", Domain Decomposition Methods for Partial Differential Equations (eds. R. Glowinski et al.), SIAM (1988) 281-300.

5. Y. Saad and M.H. Schultz, "GMRES: a Generalized Minimal Residual Algorithm for Solving Nonsymmetric Linear Systems", SIAM J. Scient. Stat. Comput., 7 (1986) 856 869.

6. T.E. Tezduyar and J. Liou, "Grouped Element-by-Element Iteration Schemes for Incompressible Flow Computations", Computer Physics Communications, 53 (1989) 441453.

7. T.E. Tezduyar, J. Liou, T. Nguyen and S. Poole, "Adaptive Implicit-Explicit and Parallel Element-by-Element Factorization Schemes", Chapter 34 in Domain Decomposition Methods (eds. T.F. Chan et al.), SIAM (1989) 443-463.

8. F. Shakib, T.J.R. Hughes and Z. Johan, "A Multi-element Group Preconditioned GMRES Algorithm for Nonsymmetric Systems Arising in Finite Element Analysis", to appear in Computer Methods in Applied Mechanics and Engineering .

9. J. Liou and T.E. Tezduyar, "Iterative Adaptive Implicit-Explicit Methods for Flow Problems", International Journal for Numerical Methods in Fluids, 11 (1990) 867-880.

10. J. Liou and T.E. Tezduyar, "A Clustered Element-by-Element Iteration Method for Finite Element Computations", Chapter 13 in Domain Decomposition Methods for Partial Differential Equations (eds. R. Glowinski et al.), SIAM (1991) 140-150.

11. J. Liou and T.E. Tezduyar, "Computation of Compressible and Incompressible Flows with the Clustered Element-by-Element Method", University of Minnesota Supercomputer Institute UMSI 90/215, October (1990).

12. T.E. Tezduyar and S. Mittal, "Finite Element Computation of Incompressible Flows", University of Minnesota Supercomputer Institute UMSI 91/152, June (1991).

13. T.E. Tezduyar, M. Behr, S.K. Aliabadi, S. Mittal and S.E. Ray, "A New Mixed Preconditioning Method for Finite Element Computations", University of Minnesota Supercomputer Institute UMSI 91/160 June (1991).

14. Y. Saad, "A Flexible Inner-Outer Preconditionned GMRES Algorithm", University of Minnesota Supercomputer Institute UMSI 91/279 November (1991).

DEPARTMENT OF AEROSPACE ENGINEERING AND MECHANICS, MINNESOTA SUPERCOMPUTER INSTITUTE AND ARMY HIGH-PERFORMANCE COMPUTING RESEARCH CENTER, UNIVERSITY OF MINNESOTA, 1200 WASHINGTON AVENUE SOUTH. MINNEAPOLIS. MN 55415 